\title{
a-bisabolol Exerts Anti-inflammatory Action and Ameliorates Collagen-induced Arthritis in Rats
}

\author{
Xi Gao ${ }^{1 \#}$, Yanli Zhang ${ }^{2 \#}$, Wentao Wang ${ }^{3}$, Zhiwen Zhang ${ }^{1}$, Chen Li $^{1}$, Hongjun Lou ${ }^{4}$
}

10.18805/IJAR.B-1395

\begin{abstract}
Background: Development of synovial hypertrophy, articular tissue inflammation and tenderness in joints is the characteristic feature of rheumatoid arthritis. Drugs that are derived from natural sources which target inflammation with reduced side-effects receive much attention. Hence, the present study was designed to investigate the anti-arthritic effects of $\alpha$-bisabolol, a natural chamomile essential oil and also an anti-inflammatory molecule on the rat model of collagen-induced arthritis (CIA).

Methods: Animal model of CIA was established and orally administered with $\alpha$-bisabolol $(100 \mathrm{mg} / \mathrm{kg})$ or vehicle and the pathological and inflammatory parameters were evaluated.

Result: The results of the present findings indicate that $\alpha$-bisabolol treatment ameliorates CIA-induced inflammation via inhibiting the proteases and inflammatory mediators suggesting that $\alpha$-bisabolol may be a potential candidate against arthritis inflammations.

Key words: $\alpha$-bisabolol, Collagen-induced arthritis, Inflammation.
\end{abstract}

\section{INTRODUCTION}

Rheumatoid arthritis (RA) is a common human autoimmune disease that has its predominant effect on the elderly and females than men. It is a chronic and systemic inflammatory disease affecting the lining of the synovial joints and can progress into disability and dependence on others for daily activities and cause social burden (Guo et al. 2018). RA projects itself in the form of swelling, redness and arthralgia that restricts the motion and instability arises. Despite these symptoms, diagnosis of RA is challenging as it involves complex blood tests and imaging which is far from reach in many of the developing countries that have a negative impact of cost, lifestyle, medical care and dietary intake. Prolonged illness can lead to keratitis, pericarditis and small vessel vasculitis and other extra-articular complications in the skin, eye, lungs, kidneys, nervous and gastrointestinal systems. Early diagnosis of RA, the effects of joint destruction, radiologic progression and functional disability can be curtailed and can be well within the reach of therapy (Heidari 2011).

The pathogenesis of RA can be in different stages and most importantly synovitis occurring in the targeting stages in small joints causing joint swelling which is the characteristic of synovial membrane inflammation due to the invasion of immune cells in them. The small joints are inflamed due to the continuous influx of leukocytes and inundation of proinflammatory mediators that results in the inflammation. Synoviocytes interact with various immune cells such as macrophages, monocytes, mast cells, DCs and $T$ and $B$-cells results in pathogenesis in RA. The production of TNF-alpha in and the expression of alphaenolase on monocytes and macrophages are the precursors to the production of proinflammatory cytokines that create the matrix rich in proinflammatory factors like IL-1 $\beta$ and IL6 cytokines (Carteron 2000) which would berth T cells and $B$ cells for further destruction of bones and cartilage in RA.
1Department of the Fourth Orthopedics, First Affiliated Hospital, Heilongjiang University of Chinese Medicine, Harbin, Heilongjiang, 150040, China.

${ }^{2}$ Experimental Teaching and Practical Training Center, Heilongjiang University of Chinese Medicine, Harbin, Heilongjiang, 150040, China. ${ }^{3}$ Department of Orthopedics of Integration TCM with Western Medicine, Heilongjiang University of Chinese Medicine, Harbin, Heilongjiang, 150040, China.

${ }^{4}$ Department of Clinical Laboratory, The First Affiliated Hospital, Heilongjiang University of Chinese Medicine, Harbin, Heilongjiang, 150040, China.

"These two authors contribute to this work equally.

Corresponding Author: Hongjun Lou, Department of Clinical Laboratory, The First Affiliated Hospital, Heilongjiang University of Chinese Medicine, Harbin, Heilongjiang, 150040, China.

Email: Ihj-hzy@163.com

How to cite this article: Gao, X., Zhang, Y., Wang, W., Zhang, Z., Li, C. and Lou, H. (2021). a-bisabolol Exerts Anti-inflammatory Action and Ameliorates Collagen-induced Arthritis in Rats. Indian Journal of Animal Research. DOI: 10.18805/IJAR.B-1395.

Submitted: 31-05-2021 Accepted: 30-07-2021 Online: 20-08-2021

The natural monocyclic sesquiterpene alcohol, $\alpha$ Bisbolol, found in the oils of some plants has been widely used as cosmetics with anti-inflammatory activities (Kim et al. 2011) and we propose this to be used as an agent in the control of pain and swelling associated with the progression of RA. The validity of our proposal can be examined by its effectiveness in controlling the lysosomal enzymes and stabilization of lysosomes in CIA in rats, which is our surrogate model of RA in humans. We studied the downstream effects of its treatment in rats by studying the impacts on the expression of various enzymes and proteases, inflammatory mediators and related mRNA in the 
progression of RA in CIA induced group along with their control groups.

\section{MATERIALS AND METHODS}

Wistar male rats weighing $150-180 \mathrm{~g}$ were used for the present study in accordance with the Institutional Animal Care and Use Committee. The experimental work was carried out in Lanzhou University Second Hospital Lanzhou from March 2018 to Dec. 2020 The experimental arthritis model was created in the rat as described earlier (Brand et al., 2007). In the present study, animals were followed up for 6 weeks with 4 groups of rats as follows, Group 1 as control with vehicle control (without collagen and adjuvant); Group 2 rats with arthritis induced, Group 3 rats administered with $\alpha$-bisabolol (100 mg/kg, p.o) for 2 weeks prior to arthritis induction and continued even after arthritis induction till the end of the experimental periods. Group 4 rats administered with $\alpha$-bisabolol alone served as drug control. For the induction of arthritis, rats were injected intradermally at the base of the tail with an emulsion (50 $\mu$ l) containing type II collagen $(3 \mathrm{mg} / \mathrm{ml})$ dissolved in $0.1 \mathrm{M}$ acetic acid at $4^{\circ} \mathrm{C}$ and Freund's complete adjuvant $(3 \mathrm{mg} / \mathrm{ml})$. After 21 days, a booster dose was given following 3 weeks after the booster dose, the animals were killed and blood was collected, plasma was separated. On the other hand, peripheral blood mononuclear cells were isolated using ficollhistopaque for qRT-PCR analysis.

To evaluate the severity of arthritis clinically, the clinical score was made in the hind paw once in a week according to the scale as follows: $0=$ Normal appearance with no evidence of erythema and swelling; 1- mild erythema and mild swelling confined to the one or several digits/tarsals or metatarsals; 2- erythema and moderate swelling of tarsal and the metatarsal or tarsal extended to tarsus; 3- erythema and severe swelling extending from ankle to metatarsal joints; and $4=$ severe inflammation, erythema and swelling encompassing the ankle, foot and digits, resulting in deformity and/or ankylosis. Scores from four limbs were calculated and the maximum score for each animal could reach was 16 . Further, the hind paw edema volume was measured using a plethysmometer by the total volume increased in a cylinder filled with buffer. Briefly, in a small cylinder filled with a buffer connected to a device capable to measure the total fluid volume, the hind paw of the animal was inserted inside the cylinder and the total volume added was then measured, the difference between the final volume minus the initial volume results to pawthe total volume and the edema between groups were also compared. In addition, grip strength and endurance exercise performance were also calculated.

For the estimation of lysosomal enzymes such as $\beta$ glucuronidase (K514-100, Biovision, Milpitas, CA), acid phosphatase (K411-500, Biovision, Milpitas, CA), $\beta$ glycosidase (BA0018, Assay Genie, Ireland), nacetylglucosaminidase (BA0053, Assay Genie, Ireland), commercial enzyme assay were used and plasma samples were tested in the kit as per the manufacturer's instruction.
In order to evaluate the levels of glycoproteins such as hexose, hexosamine, sialic acid and hexuronic acid, the plasma was separated and the colorimetric estimation was performed as described earlier(Subramanian et al., 2010).

To identify the role of proteases in the onset of arthritis, the activity of Cysteine protease cathepsin B (ab65300, Abcam, USA), dipeptidyl peptidase I (cathepsin C, ABIN829523 Antibodies online Gmbh, Germany), aspartate protease cathepsin D (ab65302, Abcam, USA) was evaluated using the commercial assay kits as per the company's instructions. For the analysis of collagen antibody raised, the ELISA method was implemented as per the company's instructions. At the end of the experiment, the levels of cytokines were analyzed using commercial kits. The levels of IL-6, IL-17, IL-18, CXC-12, IL-10, IL-1 $\beta$, IL22 were estimated per the manufacturer's instructions given in the assay kit (Fine Biotech, China).

At the end of the experimental period, total RNA was extracted from the PBMCs using TRIzol $B^{8}$ reagent. It was followed by real-time PCR analysis, the forward $(F)$ and reverse (R) primers used for the specific genes were as follows:

\section{COX-2- F:AAGGCGTTCAACTGAGCTGT; R: ACACAGGAATCTTCACAAATGGA; \\ iNOS- F:TGAAGCACTTTGGGTGACCA; R: TATACACGGAAGGGCCAAGC; \\ TNF- $\alpha$ - F: CATCCGTTCTCTACCCAGCC; R: AATTCTGAGCCCGGAGTTGG; MCP-1- F: CTGTAGCATCCACGTGCTGT, R: CTCCAGCCGACTCATTGGG; \\ GAPDH-F: AGTGCCAGCCTCGTCTCATA; R: GGTAACCAGGCGTCCGATAC.}

The gene expression was calculated from the CT values and the fold increase was determined by the comparative $\mathrm{Ct}$ method $(\triangle \Delta C T)$ with the expression values of GAPDH as an endogenous control. Statistical significance was measured with one-way analysis of variance (ANOVA) followed by a post-hoc test for multiple comparisons. For two-group comparison, student T-test was used. Differences ina $p$-value less than 0.05 were considered statistically significant.

\section{RESULTS AND DISCUSSION}

In the current investigation, a rat model of CIA was used and the protective effect of $\alpha$-bisabolol was elucidated against the onset of inflammation and precarious cell signaling activated in arthritis. The results evidenced the severity of inflammation in rats shown by the maximum score reached in the CIA group (Fig $1 \mathrm{~A}$ ) and this is explained by the increase of infiltration of immune cells into the synovium which develops into intra-articular loose bodies producing ankylosis and secondary complications of RA which is degenerative (Guo et al. 2018). The reduction in the RA score would be highlighted by the fact that $\alpha$-bisabolol has an immune protective effect in controlling the influx of immune cells and could reduce the symptoms associated with RA. Another aspect characteristic of RA induction is 
the edema in the paw of the animals (Fig 1B) which is mediated by the cytokines through capillary leakage in the acute phase of the onset of RA (Sommer et al. 2005). This leads to swelling of the synovium and the effusion exaggerates the subsequent swelling in the joints. Similar trends have been observed in our second group of rats with $\mathrm{CIA}$ and the trends were reversed in the third group which was well treated with $\alpha$-bisabolol prior to CIA treatment. Hence the symptoms of RA can be alleviated by the administration of $\alpha$-bisabolol in rats with RA.

The endurance training in rats, affected by RA, would reveal the extent of muscle loss and their functional ability which is evident in the least time clocked by the rats with RA when compared with the rats in other control and $\alpha$ bisabolol-containing groups (Fig 1C). Considerable improvement in the rats pretreated with $\alpha$-bisabolol showed that the endurance limits of this group of rats has improved and is due to lessening of the pain associated with RA and increased functional ability with decreased disease activity in them (Strasser et al. 2011). Assessing the RA symptoms' adverse effects on paws with $\alpha$-bisabolol treated animals (Fig 1D) that include measuring the grip strength(Higgins, Adams and Hughes 2018) which is improved when compared with the CIA in rats and hence the complications of the functional limitations and deformity associated with RA.

The maintenance of lysosomal membrane integrity is important in preventing RA. Acid phosphatase and $\beta$ glucuronidases levels would infer the status of lysosomal membrane integrity. Here, we observed that in CIA group, lysosomal constituents (Fig 2A, C and D) including hydrolytic enzymes degrade the glycoconjugates of the endothelial membranes and, are let into circulation that causes tissue and membrane damage (Yasuda et al. 2000). Hence, the levels of hexose, hexosamine, hexuronic acid and sialic acid are found to be increased in the CIA group (Fig 3A-D) and promote cartilage degradation and the increased bone resorption, indicates the extent of cartilage matrix degradation caused due to these enzymes (Ortutay et al. 2003) and their clinical relevance to RA. These enzymes deplete glucose aminoglycans (GAG) from cartilage and contribute to the compression of the cartilage where the GAGs otherwise provide resilience to it. The depleted GAGs would have sialic acid in their ends and could be used as a marker to diagnose the RA (Chrostek et al. 2014; Li et al. 2019)as evident in our CIA group (Fig 3D). Increased levels of sialic acid could be a possible way to defend against the biological stress associated with RA(Aryaeian et al. 2011). The normal turnover of bones after remodeling and growth mediated by acid hydrolases would be in increased levels and activity would attack the connective tissue by degrading collagen, glycoproteins and glycosaminoglycans (Sultana and Rasool 2015). These elevated attacks on RA joints would be reversed with the treatment of $\alpha$-bisabolol in CIAinduced rats. The activities of Cathepsin B, C, D and Nacetyl- $\beta$-glucosaminidase were observed to be increased in the CIA group (Fig 4A, B and C). The levels of antitype II collagen antibody levels are higher in the CIA-induced group than in other groups (Fig 4D).

The pathogenicity of RA is manifested as synovial inflammation and is augmented in CIA-induced rats by the network of proinflammatory mediators such as IL-6, IL-17, IL-18, IL-22, IL-1 $\beta$ and TNF- $\alpha$ (Choy and Panayi 2001) (Fig 5A, 5B, 5C, 5D, 5E and 6B) which are supplied by activated $T$ cells, $B$ cells and macrophages (Sultana and
A
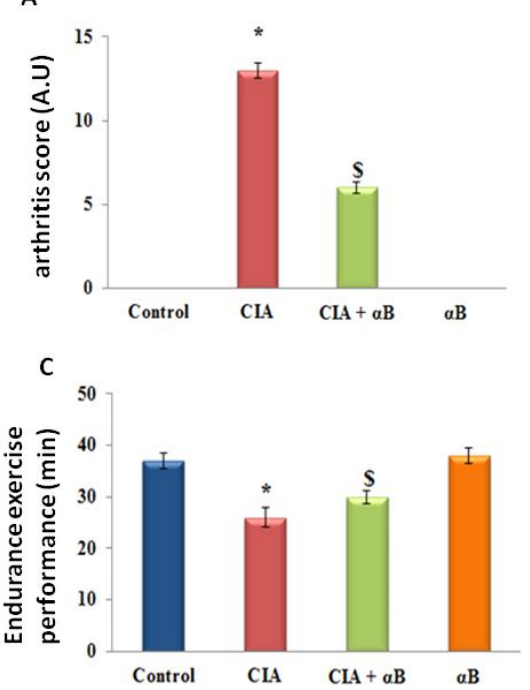

B

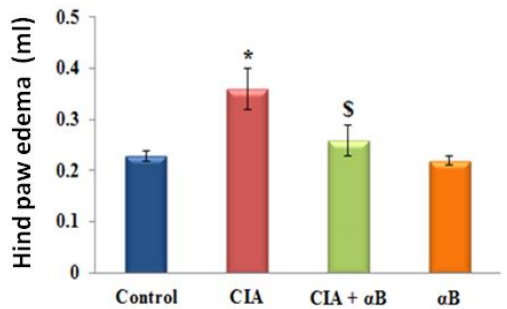

D

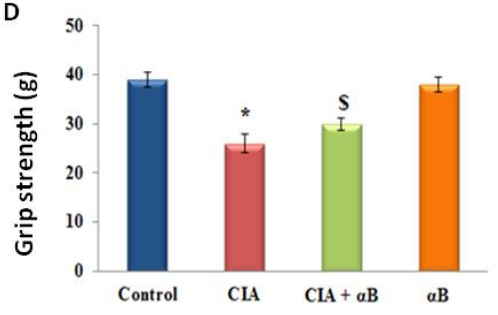

Fig 1: A-D represents the arthritis score; hind paw edema volume measured in plethysmometer; endurance exercise performance and grip strength of control and experimental group of rats respectively. Values are expressed as mean \pm S.E $(n=6)$. Statistical significance expressed as ${ }^{*} p<0.05$ of CIA compared to saline-treated controls, $\$ p<0.05$ of $\alpha$-bisabolol pre-treatment compared to CIA rats. 'ns' denotes non-significant. 

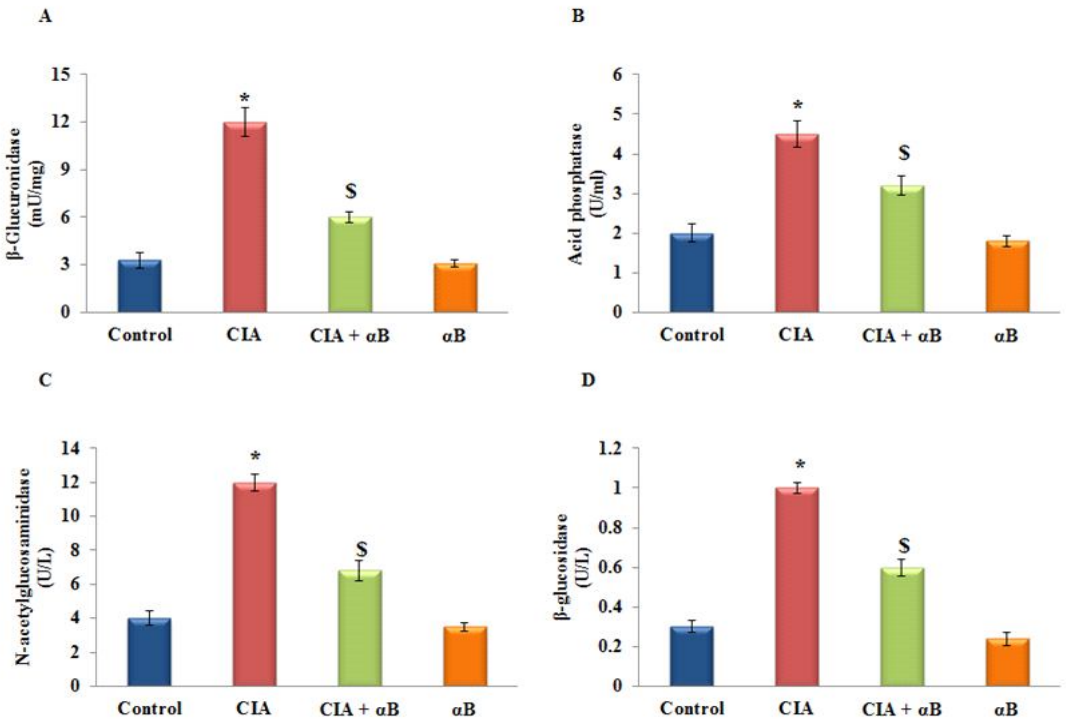

Fig 2: A-D represents the activity of $\beta$-glucuronidase, acid phosphatase, $\alpha$-glycosidase, $n$-acetylglucosaminidase in control and experimental group of rats. The units for $\beta$-glucuronidase is $\mathrm{mU} / \mathrm{mg}$; acid phosphatase is $\mathrm{U} / \mathrm{ml} ; \beta$-glycosidase is $\mathrm{U} / \mathrm{L}$ and $\mathrm{N}$ acetylglucosaminidase is $\mathrm{U} / \mathrm{L}$. Values are expressed as mean $\pm \mathrm{S}$.E $(n=6)$. Statistical significance expressed as ${ }^{*} \mathrm{p}<0.05$ of $\mathrm{CIA}$ compared to saline-treated controls, $\$ p<0.05$ of $\alpha$-bisabolol pre-treatment compared to CIA rats. 'ns' denotes non-significant.
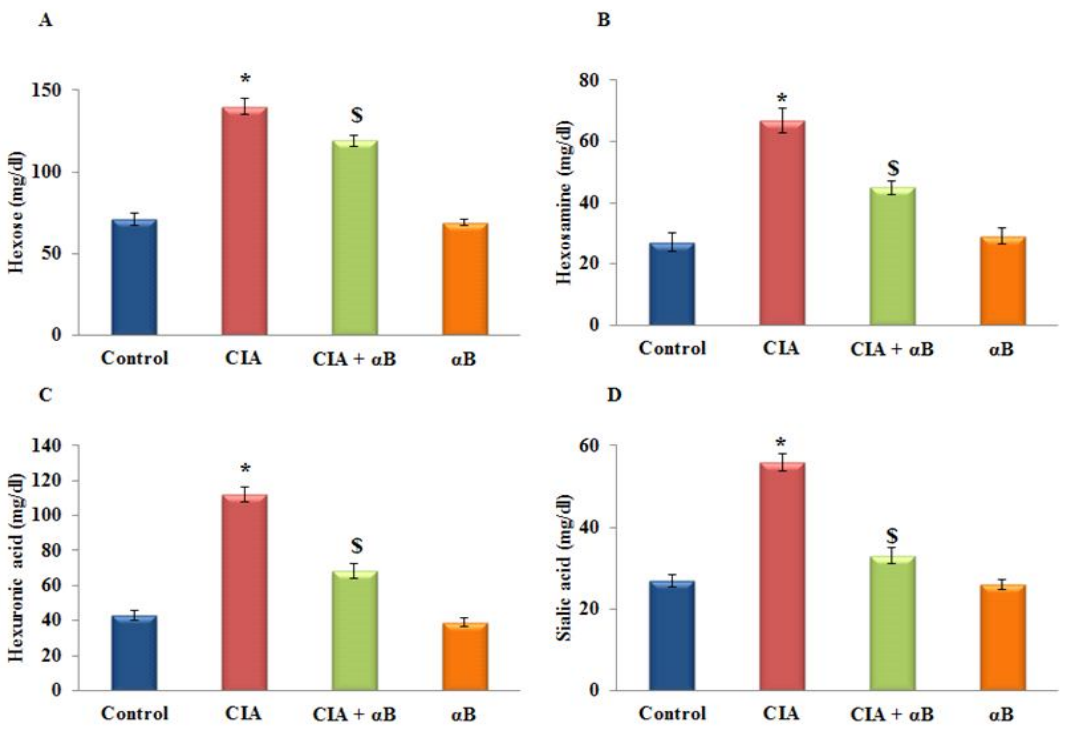

Fig 3: A-D. A-D represents the levels of glycoproteins such as hexose, hexosamine, sialic acid and hexuronic acid in control and experimental group of rats. The units for these glycoproteins is expressed as $\mathrm{mg} / \mathrm{dl}$. Values are expressed as mean $\pm S$.E $(n=6)$. Statistical significance expressed as ${ }^{*} \mathrm{p}<0.05$ of $\mathrm{CIA}$ compared to saline-treated controls, $\$ p<0.05$ of $\alpha$-bisabolol pre-treatment compared to CIA rats. 'ns' denotes non-significant.

Rasool 2015), by supplying with vascular endothelial growth factors and matrix metalloproteinases (Pan et al. 2009; Kaneko et al. 2001) to increase the infiltration of inflammatory cells into the synovial tissues. IL-17 produced by the T-cells induce the osteoclastogenesis of bone and contribute to the pathogenicity of RA with IL-22 regulating the process(Chung et al. 2006). Subsequently, the levels of expression of inducible Nitric oxide synthase (iNOS), cyclooxygenase-2 (COX2) (Lee et al. 2008; Yelin and Wanke 1999) in CIA- induced animals (Fig 6A, D) have been increased and infiltrate the RA joints and along with other lysosomal proteases and enzymes degrade the cartilage proteoglycans (Yelin and Wanke 1999) causing damage to the compressive capacity of the joints. The effects of $\alpha$-bisabolol on the inflammatory mediators such as COX-2, NO have been examined. This is evidenced by the fact that iNOS and COX-2 expression have been decreased in rats treated with our compound than in the CIA group. NO generation has been 
A

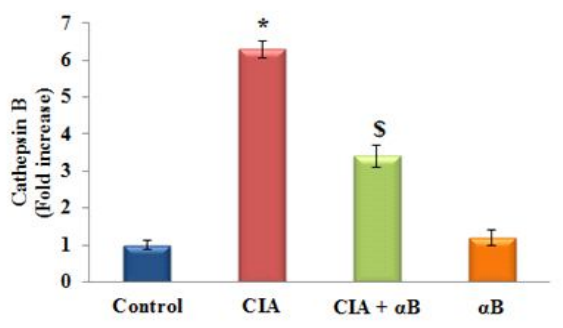

C

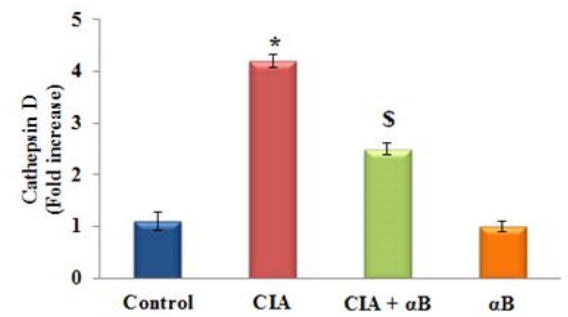

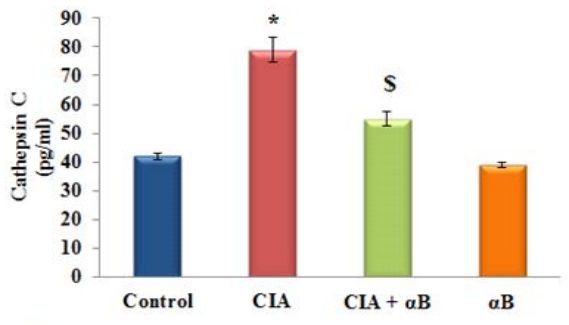

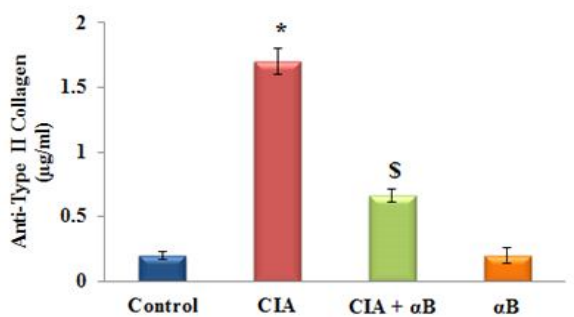

Fig 4: A-C represents the activity of Cysteine protease cathepsin B (CTSB), dipeptidyl peptidase I (CTSC), aspartate protease cathepsin $\mathrm{D}$ in control and experimental group of rats. D. Represents the anti-collagen antibody levels. Values are expressed as mean \pm S.E $(n=6)$. Statistical significance expressed as ${ }^{*} p<0.05$ of $C I A$ compared to saline-treated controls, $\$ p<0.05$ of $a$-bisabolol pre-treatment compared to CIA rats. 'ns' denotes non-significant.

A

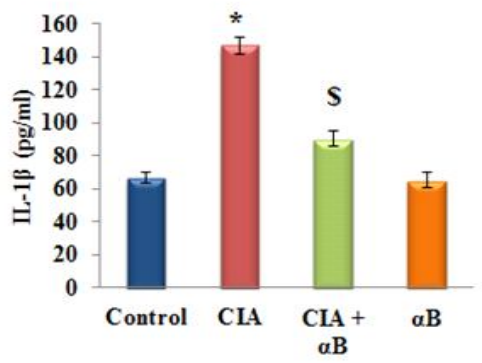

C

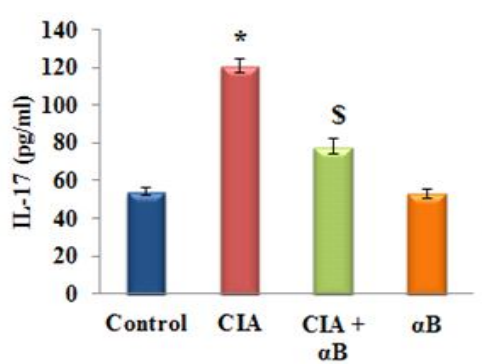

B

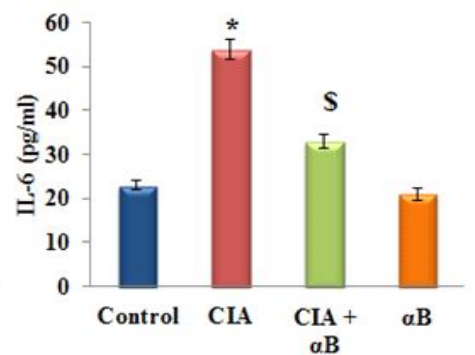

D

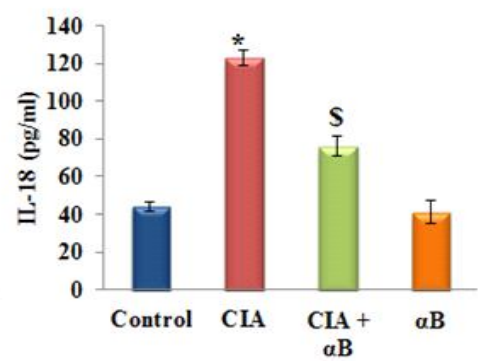

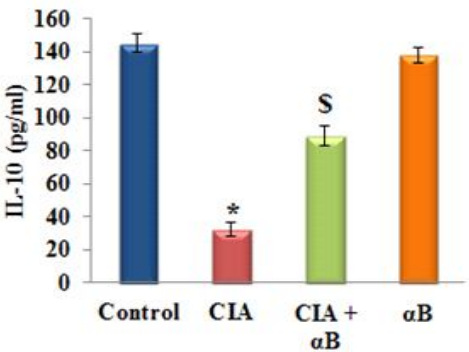

E

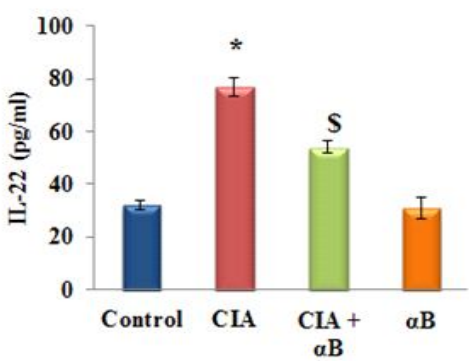

Fig 5: A-E represents cytokine expression analysis of IL-1 1 , IL-6, IL-17, IL-22, IL-18 and IL-10 in the control and experimental group of rats respectively. The detail of the experiment is given in the methodology section. Values are expressed as mean $\pm S$.E $(n=6)$.

Statistical significance expressed as ${ }^{*} \mathrm{p}<0.05$ of $\mathrm{CIA}$ compared to saline-treated controls, $\$ p<0.05$ of $\alpha$-bisabolol pre-treatment compared to CIA rats. 'ns' denotes non-significant. 

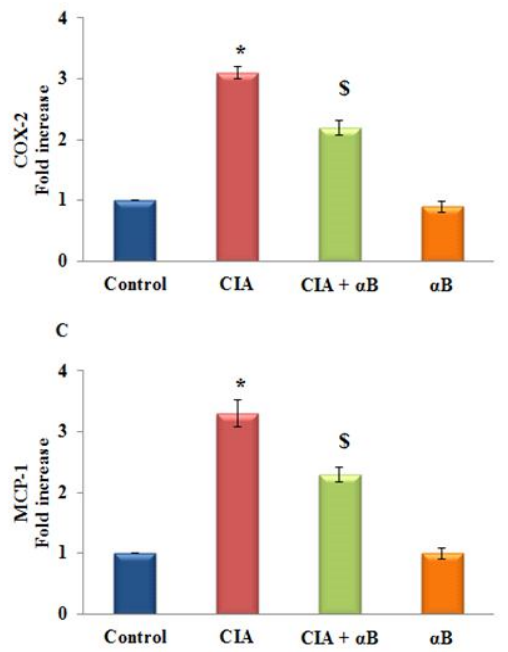

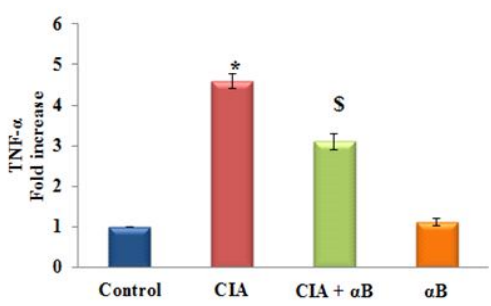

D

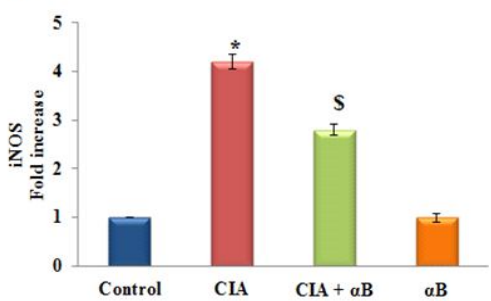

Fig 6: A-D represents qRT-PCR mRNA expression analysis of COX-2, iNOS, MCP-1, TNF- $\alpha$ in control and experimental group of rats respectively. The ezpression of mRNA is denoted as fold increase compared with the housekeeping gene GAPDH. Values are expressed as mean \pm S.E $(n=6)$. Statistical significance expressed as ${ }^{*} p<0.05$ of CIA compared to saline-treated controls,

$\$ p<0.05$ of $\alpha$-bisabolol pre-treatment compared to CIA rats. 'ns' denotes non-significant.

key to the inflammation process (Nagy et al. 2010) and is generated by iNOS whereby controlling it would be helpful in the therapeutic process in RA. In our study we have shown that CIA-induced arthritis has been reversed by $\alpha$-bisabolol and that the expression of iNOS and COX2 genes has been inhibited by exerting the anti-inflammatory effects of $\alpha$-bisabolol and thus it is an anti-inflammatory agent (Kim et al. 2011) capable of reducing the adverse effects of inflammatory mediators in CIA-induced RA.

\section{CONCLUSION}

We conclude that the inflammation in RA is due to the biochemical changes exerted by lysosomal enzymes that are released in the synovium milieu due to membrane instability and cascade recruitment of pro-inflammatory mediators to mount an immune response of macrophages, $T$ cells and $B$ cells to generate pain and swelling and subsequent disability. Our compound á-bisabolol has the anti-inflammatory properties that could stabilize the lysosome to prevent the release of its constituents and keep the pro-inflammatory activators in check to subside the proinflammatory reactions. We have a way forward in proposing the exact anti-arthritic compound rather any crude extract and detailed studies in other aspects of risks associated with its usage in RA treatment is needed.

\section{REFERENCES}

Aryaeian, N., Djalali, M., Shahram, F., Jazayeri, Sh., Chamari, M. and Nazari, S. (2011). Beta-carotene, vitamin E, MDA, glutathione reductase and arylesterase activity levels in patients with active rheumatoid rrthritis. Iran J. Public Health. 40: 102-9.
Brand, D.D., Latham, K.A. and Rosloniec, E.F. (2007). Collageninduced arthritis. Nat Protoc. 2: 1269-75.

Carteron, N.L. (2000). Cytokines in rheumatoid arthritis: trials and tribulations. Mol. Med. Today. 6: 315-23.

Choy, E.H. and Panayi, G.S. (2001). Cytokine pathways and joint inflammation in rheumatoid arthritis. N. Eng. J. Med. 344: 907-16.

Chrostek, L., Cylwik, B., Gindzienska-Sieskiewicz, E., Gruszewska, E., Szmitkowski, M. and Sierakowsk, S. (2014). Sialic acid level reflects the disturbances of glycosylation and acute-phase reaction in rheumatic diseases. Rheumatol Int. 34: 393-9.

Chung, Y., Yang, X., Chang, S.H., Ma, L., Tian, Q. and Dong, C. (2006). Expression and regulation of IL-22 in the IL-17-producing CD4+ T lymphocytes. Cell Res. 16: 902-7.

Guo, Q., Wang, Y., Xu, D., Nossent, J., Pavlos, N.J. and Xu, J. (2018). Rheumatoid arthritis: Pathological mechanisms and modern pharmacologic therapies. Bone Research. 6: 15.

Heidari, B. (2011). Rheumatoid Arthritis: Early diagnosis and treatment outcomes. Caspian Journal of Internal Medicine. 2: 16170.

Higgins, S.C., Adams, J. and Hughes, R. (2018). Measuring hand grip strength in rheumatoid arthritis. Rheumatol Int. 38: 707-714.

Kaneko, M., Tomita, T., Nakase, T., Ohsawa, Y., Seki, H., Takeuchi, E., Takano, H., Shi, K., Takahi, K., Kominami, E., Uchiyama, Y., Yoshikawa, H. and Ochi, T. (2001). Expression of proteinases and inflammatory cytokines in subchondral bone regions in the destructive joint of rheumatoid arthritis. Rheumatology (Oxford). 40: 247-55.

Kim, S., Jung, E., Kim, J.H., Park, Y.H., Lee, J. and Park, D. (2011). Inhibitory effects of (-)-alpha-bisabolol on LPS-induced inflammatory response in RAW264.7 macrophages. Food Chem. Toxicol. 49: 2580-5. 
Lee, S.J., Nam, W.D., Na, H.J., Cho, Y.L., Ha, K.S., Hwang, J.Y., Lee, H., Kim, S.O., Kwon, Y.G. and Kim, Y.M. (2008). CT20126, a novel immunosuppressant, prevents collageninduced arthritis through the down-regulation of inflammatory gene expression by inhibiting NF-kappaB activation. Biochem Pharmacol. 76: 79-90.

Li, W., Liu, Y., Zheng, X., Gao, J., Wang, L. and Li, Y. (2019). Investigation of the potential use of sialic acid as a biomarker for rheumatoid arthritis. Ann. Clin. Lab. Sci. 49: 224-231.

Nagy, G., Koncz, A., Telarico, T., Fernandez, D., Ersek, B., Buzas, E. and Perl, A. (2010). Central role of nitric oxide in the pathogenesis of rheumatoid arthritis and systemic lupus erythematosus. Arthritis Res. Ther. 12: 210.

Ortutay, Z., Polgar, A., Gomor, B., Geher, P., Lakatos, T., Glant, T.T., Gay, R.E., Gay, S., Pallinger, E., Farkas, C., Farkas, E., Tothfalusi, L., Kocsis, K., Falus, A. and Buzas, E.I. (2003). Synovial fluid exoglycosidases are predictors of rheumatoid arthritis and are effective in cartilage glycosaminoglycan depletion. Arthritis Rheum. 48: 2163-72.

Pan, R., Y. Dai, X. Gao and Xia, Y. (2009). Scopolin isolated from Erycibe obtusifolia Benth stems suppresses adjuvantinduced rat arthritis by inhibiting inflammation and angiogenesis. Int. Immunopharmacol. 9: 859-69.

Sommer, O.J., Kladosek, A., Weiler, V., Czembirek, H., Boeck, M. and Stiskal, M. (2005). Rheumatoid arthritis: A practical guide to state-of-the-art imaging, image interpretation and clinical implications. Radiographics. 25: 381-98.
Strasser, B., Leeb, G., Strehblow, C., Schobersberger, W., Haber, P. and Cauza, E. (2011). The effects of strength and endurance training in patients with rheumatoid arthritis. Clin. Rheumatol. 30: 623-32.

Subramanian, U., Poongavanam, S. and. Vanisree, A.J (2010). Studies on the neuroprotective role of Piper longum in C6 glioma induced rats. Invest New Drugs. 28: 61523.

Sultana, F. and Rasool, M. (2015). A novel therapeutic approach targeting rheumatoid arthritis by combined administration of morin, a dietary flavanol and non-steroidal antiinflammatory drug indomethacin with reference to proinflammatory cytokines, inflammatory enzymes, RANKL and transcription factors. Chem. Biol. Interact. 230: 5870.

Yasuda, M., Okabe, T., Itoh, J., Takekoshi, S., Hasegawa, H., Nagata, H., Osamura, R.Y. and Watanab, K. (2000). Differentiation of necrotic cell death with or without Iysosomal activation: application of acute liver injury models induced by carbon tetrachloride (CCL4) and dimethylnitrosamine (DMN). J. Histochem Cytochem. 48: 1331-9.

Yelin, E. and Wanke, L.A. (1999). An assessment of the annual and long-term direct costs of rheumatoid arthritis: The impact of poor function and functional decline. Arthritis Rheum. 42: 1209-18. 